\title{
Dexmedetomidine inhibits astrocyte pyroptosis and subsequently protects the brain in in vitro and in vivo models of sepsis
}

\author{
Yi-Bing Sun ${ }^{1}$, Hailin Zhao ${ }^{2}$, Dong-Liang Mu', Wenwen Zhang ${ }^{2,3}$, Jiang Cuil, Lingzhi Wư ${ }^{2}$ Azeem Alam², \\ Dong-Xin Wang ${ }^{1}$ and Daqing Ma $\mathbb{B}^{2}$
}

\begin{abstract}
Sepsis is life-threatening and often leads to acute brain damage. Dexmedetomidine, an $\mathrm{a}_{2}$-adrenoceptor agonist, has been reported to possess neuroprotective effects against various brain injury but underlying mechanisms remain elusive. In this study, in vitro and in vivo models of sepsis were used to explore the effects of dexmedetomidine on the inflammasome activity and its associated glia pyroptosis and neuronal death. In vitro, inflammasome activation and pyroptosis were found in astrocytes following lipopolysaccharide (LPS) exposure. Dexmedetomidine significantly alleviated astrocyte pyroptosis and inhibited histone release induced by LPS. In vivo, LPS treatment in rats promoted caspase-1 immunoreactivity in astrocytes and caused an increase in the release of pro-inflammatory cytokines of IL-1 $\beta$ and IL-18, resulting in neuronal injury, which was attenuated by dexmedetomidine; this neuroprotective effect was abolished by $\mathrm{a}_{2}{ }^{-}$ adrenoceptor antagonist atipamezole. Dexmedetomidine significantly reduced the high mortality rate caused by LPS challenge. Our data demonstrated that dexmedetomidine may protect glia cells via reducing pyroptosis and subsequently protect neurons, all of which may preserve brain function and ultimately improve the outcome in sepsis.
\end{abstract}

\section{Introduction}

Sepsis, a severe systemic inflammatory response as a result of infection, is associated with high morbidity and mortality rates. It is the most common reason for admission to an intensive care unit and is one of the leading causes of death. Sepsis-induced brain injury occurs in the early stage of sepsis in critically ill patients, and contributes to the progression of sepsis ${ }^{1}$. Patients with sepsis-induced brain injury often have changes

\footnotetext{
Correspondence: Dong-Xin Wang (wangdongxin@hotmail.com) or Daqing Ma (d.ma@imperial.ac.uk)

'Department of Anesthesiology and Critical Care Medicine, Peking University First Hospital, Beijing, China

${ }^{2}$ Anaesthetics, Pain Medicine and Intensive Care, Department of Surgery and Cancer, Faculty of Medicine, Imperial College London, Chelsea and Westminster Hospital, London, UK

Full list of author information is available at the end of the article. Edited by A. Verkhrtasky
}

in mental state, ranging from delirium to coma, accompanied by altered levels of consciousness, cognition, and perceptual abnormalities. Postmortem analyses demonstrate that the pathophysiology of sepsis-induced brain injury often shows diffuse brain microabscesses, indicating that the injury is likely associated with bacterial and/or endotoxin direct invasion to the brain ${ }^{2}$. Growing evidence indicates that this process involves inflammatory mechanisms leading to the central nervous system (CNS) dysfunction $^{3,4}$, neuroinflammation and related excitotoxicity are involved in the pathological process of neurological dysfunction induced by sepsis. In addition, astrocytes play a critical role in providing functional support for neurons and regulate the communication with adjacent neurons via gliotransmission, synaptic plasticity, gap junctions, and energy metabolic support ${ }^{5}$. Furthermore, astrocytes participate in brain injury associated with

\section{(c) The Author(s) 2019}

(c) (i) Open Access This article is licensed under a Creative Commons Attribution 4.0 International License, which permits use, sharing, adaptation, distribution and reproduction cc. in any medium or format, as long as you give appropriate credit to the original author(s) and the source, provide a link to the Creative Commons license, and indicate if changes were made. The images or other third party material in this article are included in the article's Creative Commons license, unless indicated otherwise in a credit line to the material. If material is not included in the article's Creative Commons license and your intended use is not permitted by statutory regulation or exceeds the permitted use, you will need to obtain permission directly from the copyright holder. To view a copy of this license, visit http://creativecommons.org/licenses/by/4.0/. 
neuroinflammation through the release of proinflammatory cytokines and toxic molecules, whilst the activation of astrocytes results in detrimental effects on neuronal function and can cause deleterious neurological sequalae $e^{6,7}$. Therefore, the protection of astrocytes could be a potential therapeutic target against sepsis-induced brain injury.

Dexmedetomidine, an $\alpha_{2}$-adrenoceptor agonist, is widely used in the perioperative period for critically ill patients in the intensive care unit for sedation, analgesia, and anxiolysis. A previous study showed that patients treated with dexmedetomidine had a greater number of ventilator-free hours than the placebo-treated group ${ }^{8}$. It has been even suggested that the choice of dexmedetomidine rather than a benzodiazepine is strongly recommended for preventing delirium in critically ill patients ${ }^{9}$. Recent evidence also suggests that dexmedetomidine increases survival up to 2 years for elderly patients admitted to ICU, as well as improves cognitive function and quality of life in 3-year survivors ${ }^{10}$. In addition, protective effects of dexmedetomidine have been reported against apoptosis, necrosis, and autophagy in the brain and peripheral tissues in various in vitro and in vivo models ${ }^{11-13}$. Dexmedetomidine has been shown to provide protection against bilirubin-induced lung damage by inhibiting cell apoptosis and promoting cell survival ${ }^{12}$. In addition, dexmedetomidine-mediated protection against brain injury induced by ischemia-reperfusion injury has been demonstrated, which is thought to be mediated by autophagy suppression ${ }^{13}$. However, whether dexmedetomidine can inhibit pyroptosis in the central nervous system in sepsis setting remains unknown.

Pyroptosis is a pro-inflammatory form of cell death and a regulated form of necrosis, distinct from other forms of cell death associated with inflammasome activation. The inflammasome functions as a sensor to detect invading pathogens and cellular damage danger signals, and induces the excessive production of potent pro-inflammatory cytokines, which augments inflammation-mediated organ injury ${ }^{14}$. Cells undergoing pyroptosis experience cellular swelling, rapid membrane rupture, DNA fragmentation, and extravasation of their intracellular contents, resulting in toxicity to adjacent healthy cells and causing further cell death. Pyroptosis can be triggered by various pathological stimuli, such as microbial infection and ischemia reperfusion ${ }^{15,16}$. Accumulating evidence has demonstrated that this novel type of cell death may play a critical role in the pathogenesis of central nervous system diseases, including Alzheimer's disease and multiple sclerosis, which are characterized by neuronal damage and neuroinflammation ${ }^{17}$. Furthermore, it has been found that a range of cell types in the CNS may undergo inflammasome-induced pyroptosis ${ }^{18,19}$. These cells, including microglia and astrocytes, exhibit NLRP3 inflammasome-related responses, which may be responsible for the deterioration in neurologic function $^{18,19}$. Studies have demonstrated that dexmedetomidine may alleviate the inflammatory response by reducing inflammasome activation in the liver and pancreas $^{20,21}$; however, the anti-inflammatory effects of dexmedetomidine associated with pyroptosis in sepsisinduced brain injury require further investigation. In this study, we set out to evaluate the hypothesis that dexmedetomidine may protect the brain via inhibiting glia pyroptosis in in vitro and in vivo models of sepsis.

\section{Results}

\section{LPS but not TNF-a induces NLRP3 inflammasome activation and pyroptosis in cultured astrocytes}

To explore the cytotoxicity of pathogenic mediators of sepsis, the $1321 \mathrm{~N} 1$ astrocytes were cultured with LPS or TNF- $\alpha$ as an extrinsic and intrinsic mediator, respectively, followed by propidium iodide (PI) staining and flow cytometry assessment. Both LPS and TNF- $\alpha$ caused cell death in a dose-dependent manner $(p<0.01)$ (Fig. 1a-d). Further, the pyroptosis-related proteins were assessed. As shown in the western blot analysis, LPS treatment stimulated the upregulation of NLRP3 (nucleotide-binding domain, leucine-rich repeat containing protein 3), ASC (apoptosis-associated speck-like protein containing a CARD), caspase-1 and GSDMD (gasdermin D) expression $(p<0.01$, Fig. 1e, g). Those proteins were not increased in the TNF- $\alpha$ cultured astrocytes $(p>0.05$, Fig. 1f, h), indicating that caspase- 1 activation and pyroptosis occurred with LPS, but not TNF-induced astrocyte cell death.

\section{Dexmedetomidine attenuates LPS-induced pyroptosis in cultured astrocytes}

Dexmedetomidine was found to be cytoprotective against LPS-induced pyroptosis (Fig. 2). Astrocytes treated with dexmedetomidine demonstrated a significant reduction in the PI-positive cells by $34 \%(p<0.01$, Fig. $2 \mathrm{a}$, b) in comparison with that in the LPS group. The expression of ASC and caspase-1 co-localization was evident in cultured astrocytes after exposure to LPS. In addition, the redistribution of ASC protein from nuclei to cytoplasm indicated inflammasome activation, while dexmedetomidine treatment resulted in astrocytes returning to a state similar to the naive control group (Fig. 2c). In addition, dexmedetomidine significantly reduced the expression of NLRP3 by $25 \%(p<$ 0.05 , Fig. $2 \mathrm{e}$ ), ASC by $32 \%$ ( $p<0.05$, Fig. $2 \mathrm{f})$, caspase- 1 by $34 \%(p<0.01$, Fig. $2 \mathrm{~g})$, and GSDMD by $30 \%(p<0.01$, Fig. $2 \mathrm{~h}$ ) relative to the control. Taken together, these findings indicate that dexmedetomidine ameliorates NLRP3 inflammasome recruitment and caspase- 1 activation, as well as decreases pyroptosis in LPS-induced astrocyte injury. 


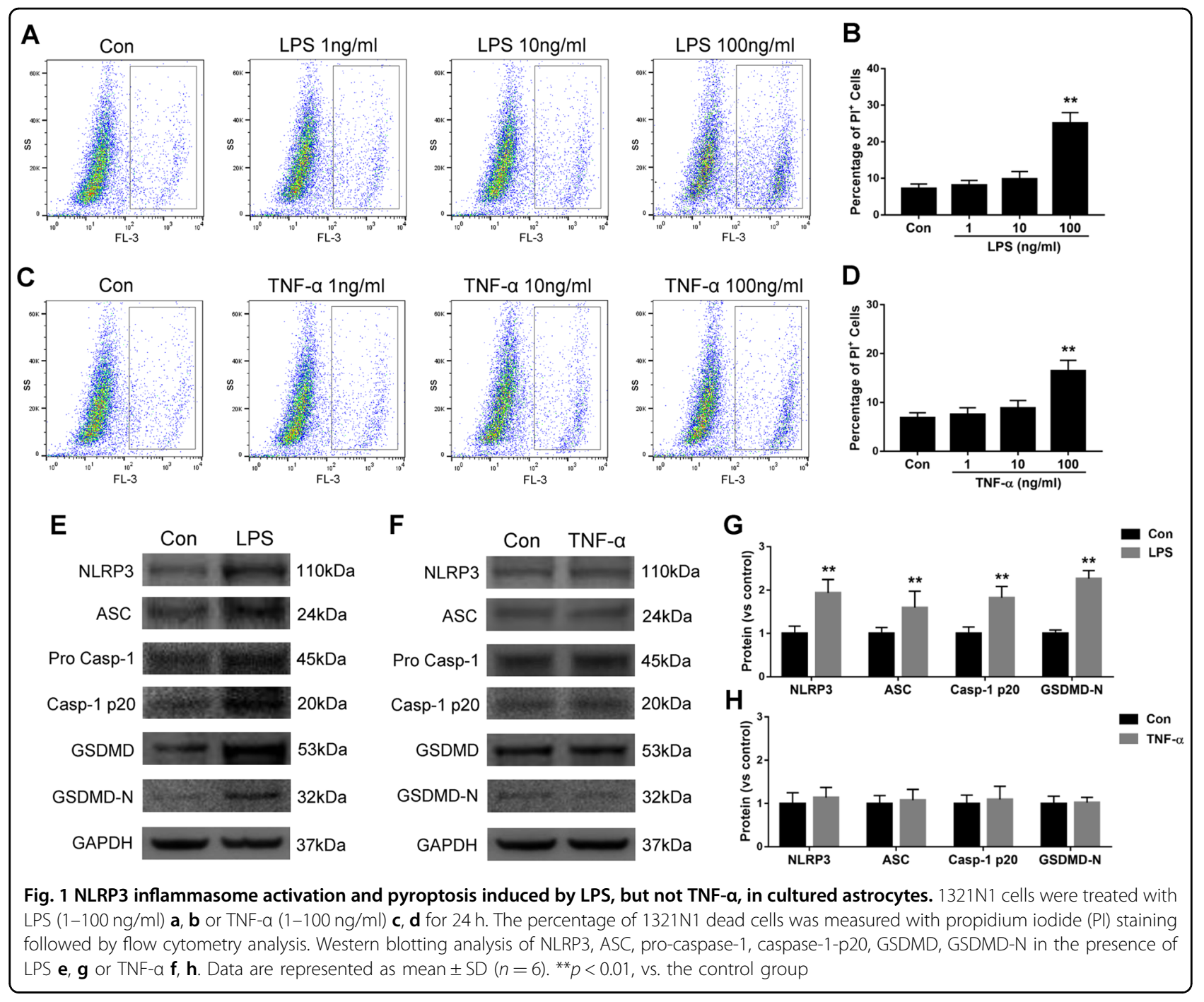

\section{Dexmedetomidine decreases histone release after LPS exposure in cultured astrocytes}

Nucleus protein histones can be released to the extracellular space from dying cells during cell injury with enhanced destruction of cellular components. A high level of histone in the cytoplasm was detected after astrocytes were challenged with LPS and demonstrated a more chaotic distribution of cytoskeleton structure in contrast to controls. With dexmedetomidine treatment, histone was restricted, to a large extent, to the nucleus and the cellular integral structure was also preserved $(p<$ 0.01 , Fig. 3a-c). Quantification of histone in the culture medium of LPS-treated cells revealed a significant increase in the release of histone compared to untreated cells; dexmedetomidine markedly inhibited histone release after LPS challenge $(p<0.01$, Fig. $3 d)$. These results indicate that dexmedetomidine prevents the disruption of the plasma membrane and maintains cellular morphology.
Recombinant histone protein induces neurotoxicity in cultured neuronal like cells

Extracellular histones are cytotoxic and may damage surrounding milieu during particular types of cell death processes, including pyroptosis. To evaluate the role of endogenously produced histones and their effect on surrounding neurons, we administrated exogenous histones to PC12 cells. As indicated in Fig. 4, toxicity mediated by histones was evaluated by double fluorescent staining with Hoechst 33342 and PI. The number of live cells (Hoechst 33342 normal/PI negative), apoptotic cells (Hoechst 33342 positive/PI negative) and necrotic cells (PI positive) was determined (Fig. 4a). Hoechst and PI staining showed cell death after histone treatment in a dose-dependent manner $(p<0.01$, Fig. $4 \mathrm{c})$. The morphological pattern of PC12 cells was significantly changed after histone stimulation (Fig. 4b). The cells exhibited small and round cell bodies and short arborizations in comparison with that in the control group, which displayed long distal 


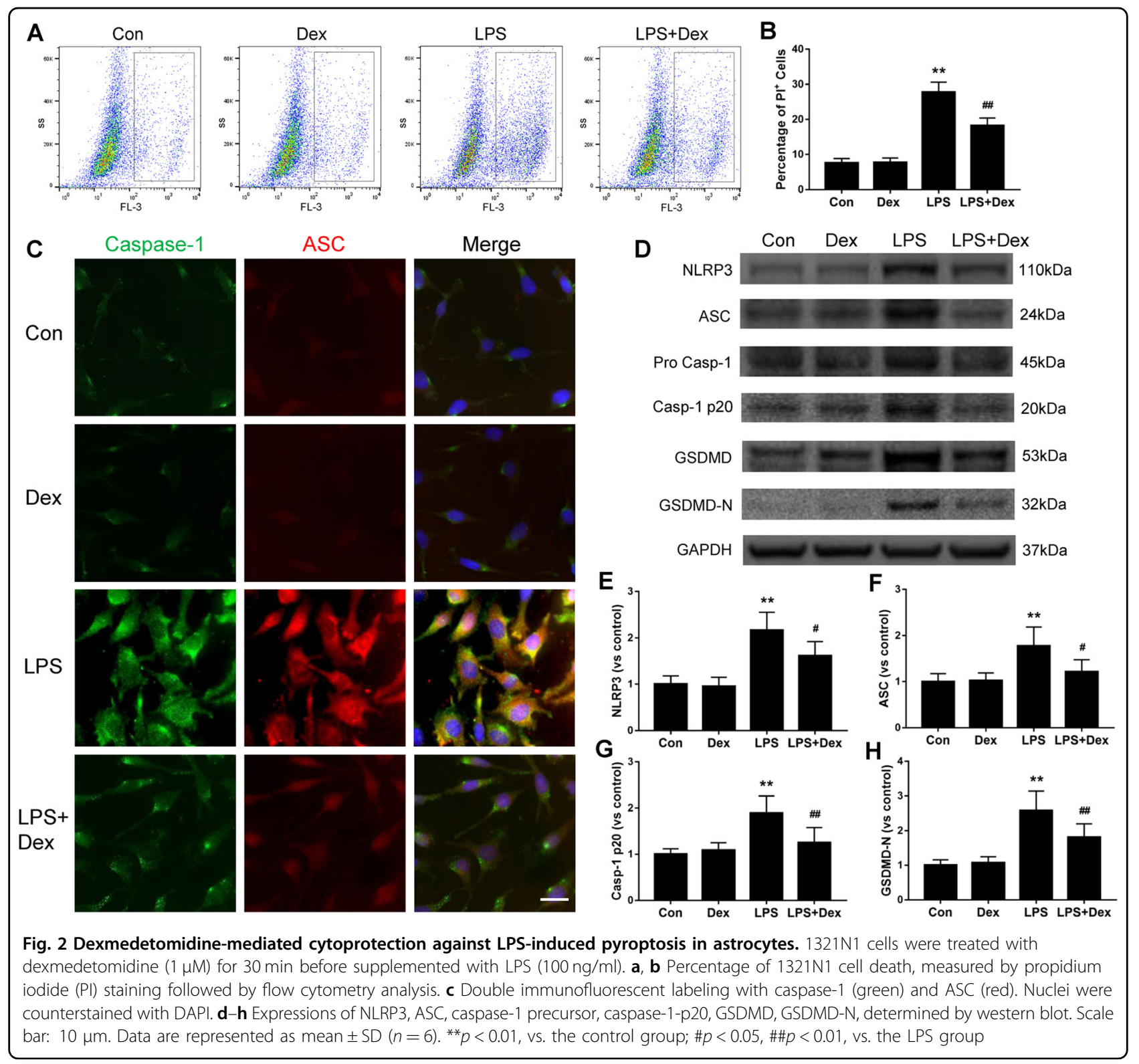

arborization. Histones, thus, are able to cause neuronal like cell damage following both low-dose and high-dose histone administration.

\section{Dexmedetomidine mediates neuroprotection against} astrocyte pyroptosis and neuroinflammation in septic rats

To further confirm our in vitro findings, the effects of dexmedetomidine on brain protection were also examined in vivo. Double immunofluorescent staining of caspase-1/GFAP was performed. LPS treatment increased the expression of caspase- 1 activation and resulted in the co-localization of caspase-1 with GFAPpositive cells in the hippocampus of septic rats, when compared with the untreated animals. In contrast, caspase-1 activation was weakly expressed in astrocytes in the dexmedetomidine group compared with that in the LPS group $(5.8 \pm 1.3 \%$ vs. $9.1 \pm 1.8 \%, p<0.01$, Fig. 5a, b). The $\alpha_{2}$-adrenoceptor antagonist, atipamezole, blocked the effect of dexmedetomidine-induced caspase-1/GFAP reducing expression $(8.9 \pm 1.8 \%$ vs. $5.8 \pm 1.3 \%, p<0.01$, Fig. $5 \mathrm{a}, \mathrm{b})$. IL- $1 \beta$, IL-18, and histone secretion were also determined in the hippocampus of the rats, which were dramatically elevated in the LPS group, compared to the control group. Dexmedetomidine caused a significant reduction in the production of pyroptosis-related cytokines, IL-1 $\beta$ $(110.19 \pm 24.12$ vs. $164.38 \pm 35.32, p<0.01$, Fig. 5 c $)$ and IL-18 $(35.25 \pm 8.67$ vs. $47.22 \pm 9.26, p<0.05$, 


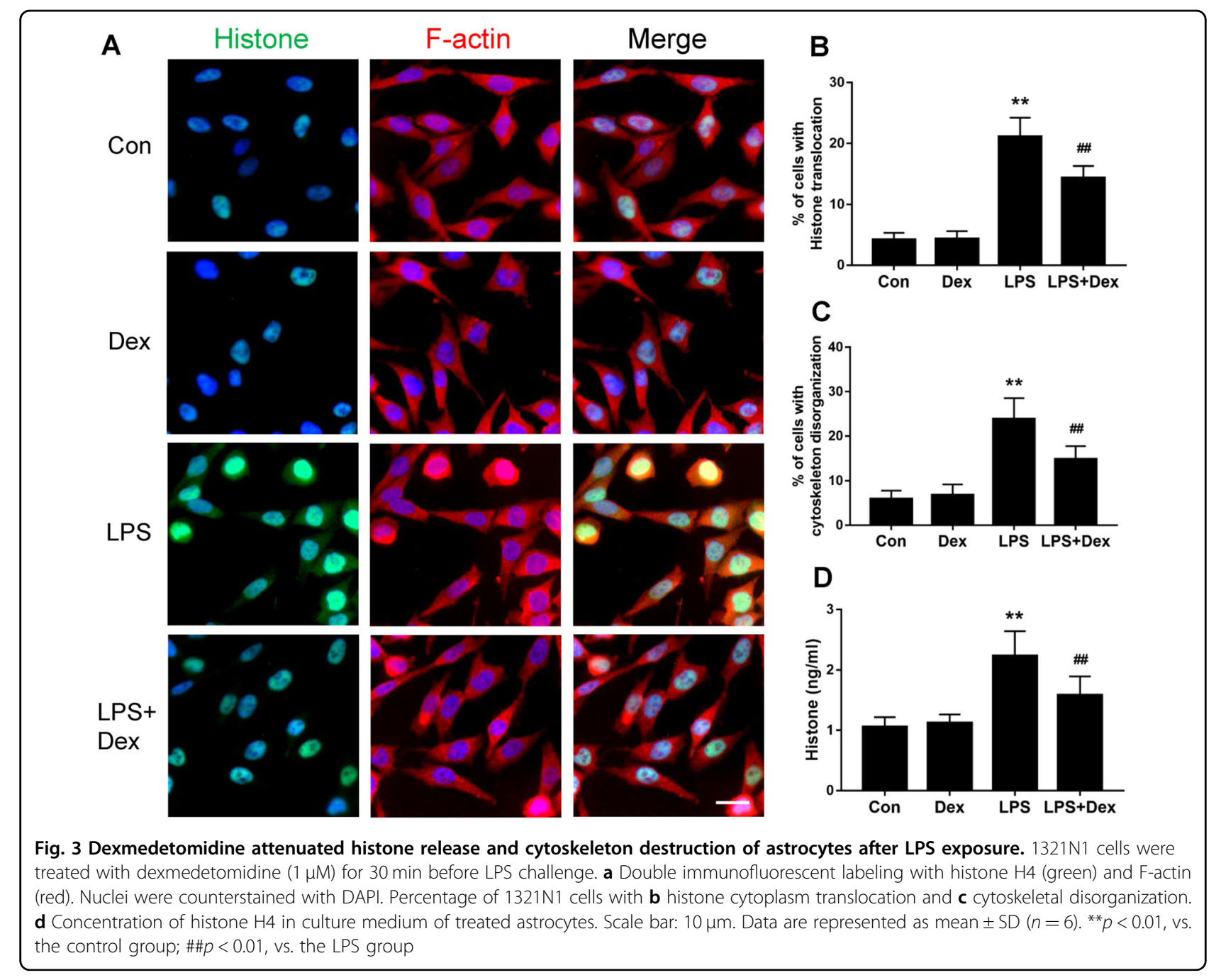

Fig. $5 \mathrm{~d}$ ). The reduction in the concentration of histone suggested an attenuation of histone release after dexmedetomidine administration $(0.07 \pm 0.01$ vs. $0.1 \pm$ $0.02, p<0.01$, Fig. 5e). Hippocampal neuronal cell injury was determined by caspase- $3 / \mathrm{NeuN}$ immunostaining in tissue sections. Compared to the control group, an increased accumulation of caspase- 3 and an increase in the number of caspase-3-positive neuronal cells (NeuN-positive cells) were also observed in the LPS-challenged rats. The neuroprotective effect of dexmedetomidine was found by suppressing caspase-3/ NeuN expression $(16.4 \pm 4.0 \%$ vs. $27.2 \pm 3.7 \%, p<0.01$, Fig. 6a, b) whilst atipamezole abolished the caspase-3/ NeuN expression downregulation mediated by dexmedetomidine $(24.3 \pm 3.2 \%$ vs. $16.4 \pm 4.0 \%, p<0.01$, Fig. 6a, b). Collectively, these findings indicate that dexmedetomidine treatment results in a decrease in neuroinflammation, pyroptosis-associated neuron damage, and histone release in a sepsis model of brain injury (Fig. 6c).
Dexmedetomidine improves survival in septic rats

Rats exhibited severe sepsis behavior and had a high mortality with LPS injection during the experimental duration of $72 \mathrm{~h}$. Dexmedetomidine significantly increased the survival rate when compared to that in the LPS-treated group (72.22\% vs. $33.33 \%, p=0.0125$, Fig. 7). Atipamezole decreased the protective effect of dexmedetomidine (38.89\% vs. $72.22 \%, p=0.0362$, Fig. 7).

\section{Discussion}

Studies have demonstrated that dexmedetomidine exhibits neuroprotective effects in various disease models $^{22-24}$; however, the neuroprotective role of dexmedetomidine against a novel form of cell death, pyroptosis, in sepsis-induced brain injury is still unknown. In the present study, we demonstrated that dexmedetomidine exerted brain protection in a model of sepsis in vitro and in vivo. Dexmedetomidine protected brain/neurons by ameliorating astrocyte pyroptosis and harmful neuroinflammation, and improved survival following LPS 


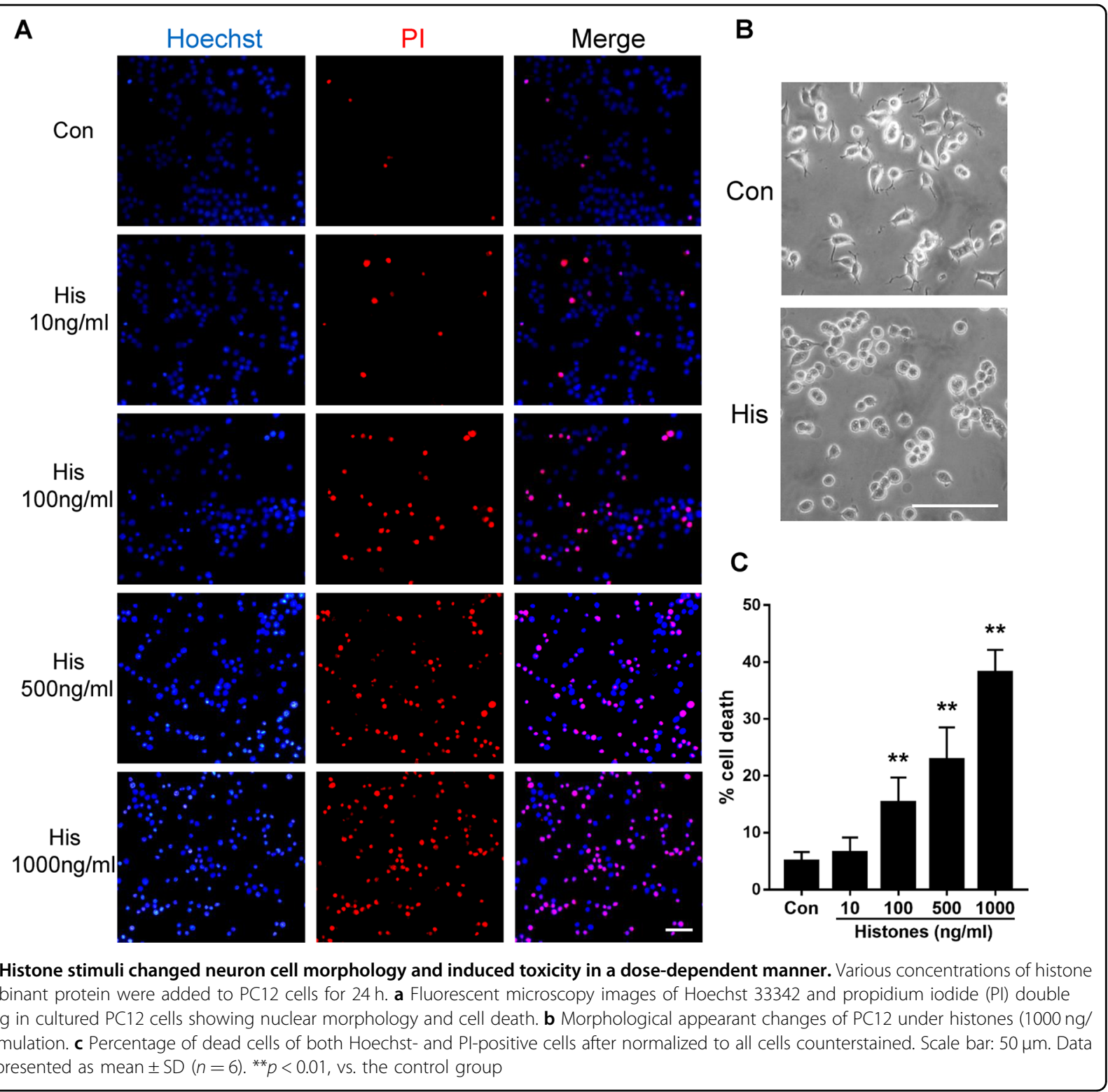

challenge. Its neuroprotective effect was abolished by $\alpha_{2}$ adrenoceptor antagonist, indicating that the dexmedetomidine provided neuroprotection likely via $\alpha_{2}$-adrenoceptor activation.

During sepsis, innate immune cells are activated by foreign molecular products known as pathogen-associated molecular patterns (PAMPs) molecules, resulting in the activation of inflammatory cells and an increase in proinflammatory cytokines ${ }^{25}$. Furthermore, various forms of cell death are involved in sepsis ${ }^{26-29}$. In this study, we chose LPS and TNF- $\alpha$ as a typical example of an endotoxin produced by Gram-negative bacteria, and a main mediator of pro-inflammatory cytokines release during sepsis, respectively. We found that both were able to cause astrocyte death. However, inflammasome activation and GSDMD cleavage were observed in the LPS group, whilst neither of them was appeared in response to TNF$\alpha$, indicating LPS but not TNF- $\alpha$ stimuli induced astrocyte pyroptosis during the cell death process.

Pyroptosis, an inflammatory form of programmed cell death, is stimulated by a broad range of pathogens. Driven by inflammasome activation, pyroptosis may contribute to the development of sepsis and septic shock ${ }^{30}$. During pyroptosis, the inflammasome complex is activated and assembled, facilitating caspase- 1 activation ${ }^{14,31}$. Subsequently, the inflammatory caspase promotes the cleavage of gasdermin D (GSDMD) protein, which induces pore formation in the plasma membrane, resulting in 


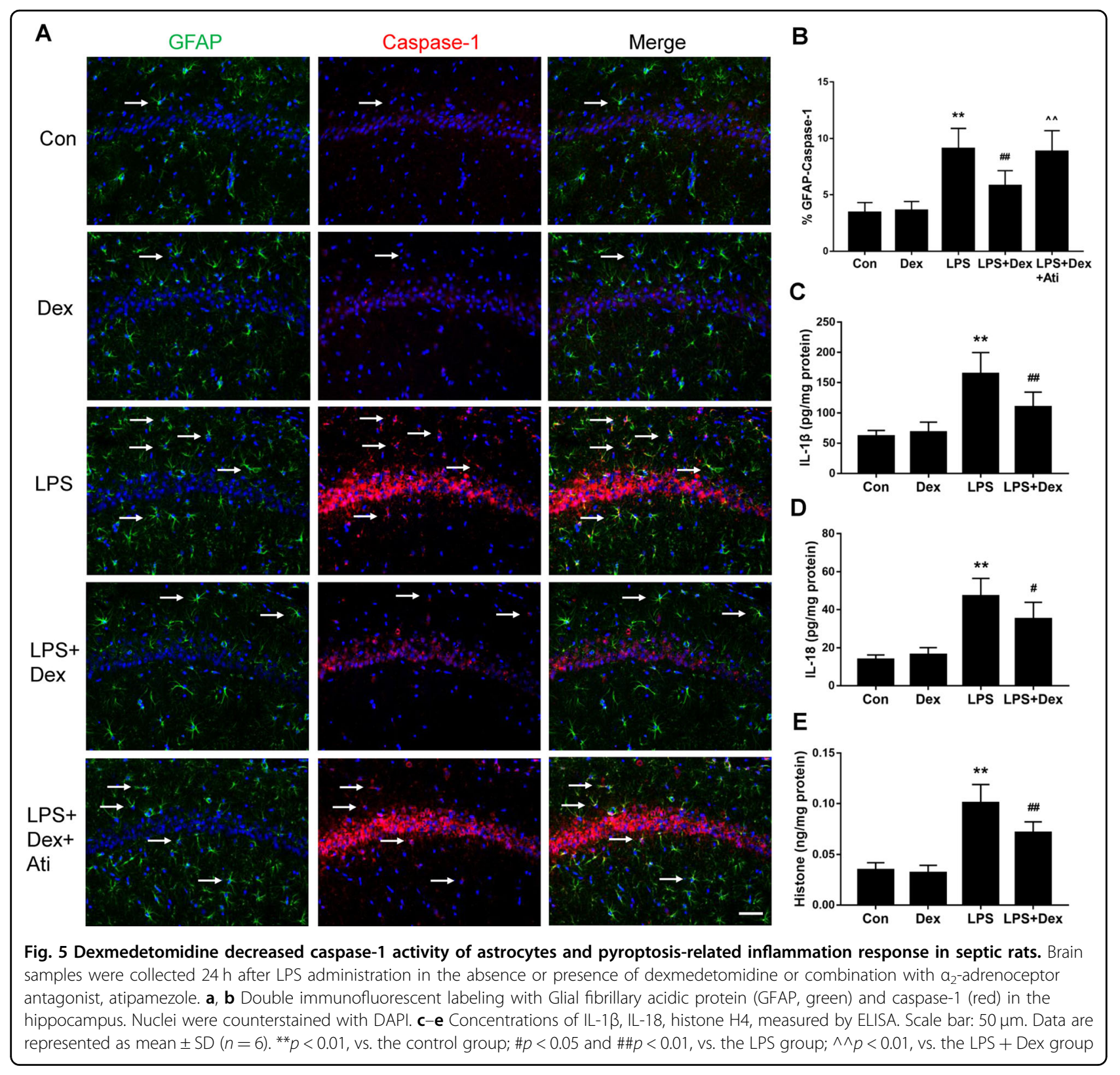

pyroptosis-induced lytic cell death ${ }^{32,33}$. Among the inflammasome complexes, the NLRP3 inflammasome is the most frequently characterized and has demonstrated a crucial role within CNS. The NLRP3 inflammasome complex is formed following the recruitment of ASC and pro-caspase-1, resulting in inflammasome activation and the initiation of pyroptosis ${ }^{30}$. In addition, pyroptosis has been implicated in the pathogenesis of several neurological diseases, including Alzheimer's disease, traumatic brain injury, and epilepsy ${ }^{17,18}$.

In this study, the NLRP3 inflammasome and caspase-1 activation were increased in astrocyte cultures treated with LPS. Following NLRP3 inflammasome assembly and activation of its downstream signaling pathways, we observed swollen astrocytes and a significant increase in the cleavage of GSDMD following LPS treatment, thus confirming activation of the pyroptotic death pathway. One of the features of pyroptosis is pore formation in the cell membrane, which can be induced by GSDMD cleavage $^{32,33}$, resulting in rupture of the plasma membrane and release of cytosolic cellular contents including intranuclear proteins ${ }^{34,35}$. Histones are intranuclear proteins that provide structural stability to nuclear chromatin and regulate gene transcription. Furthermore, histones may be released into the extracellular space from dying cells, particularly after necrosis including necroptosis, 


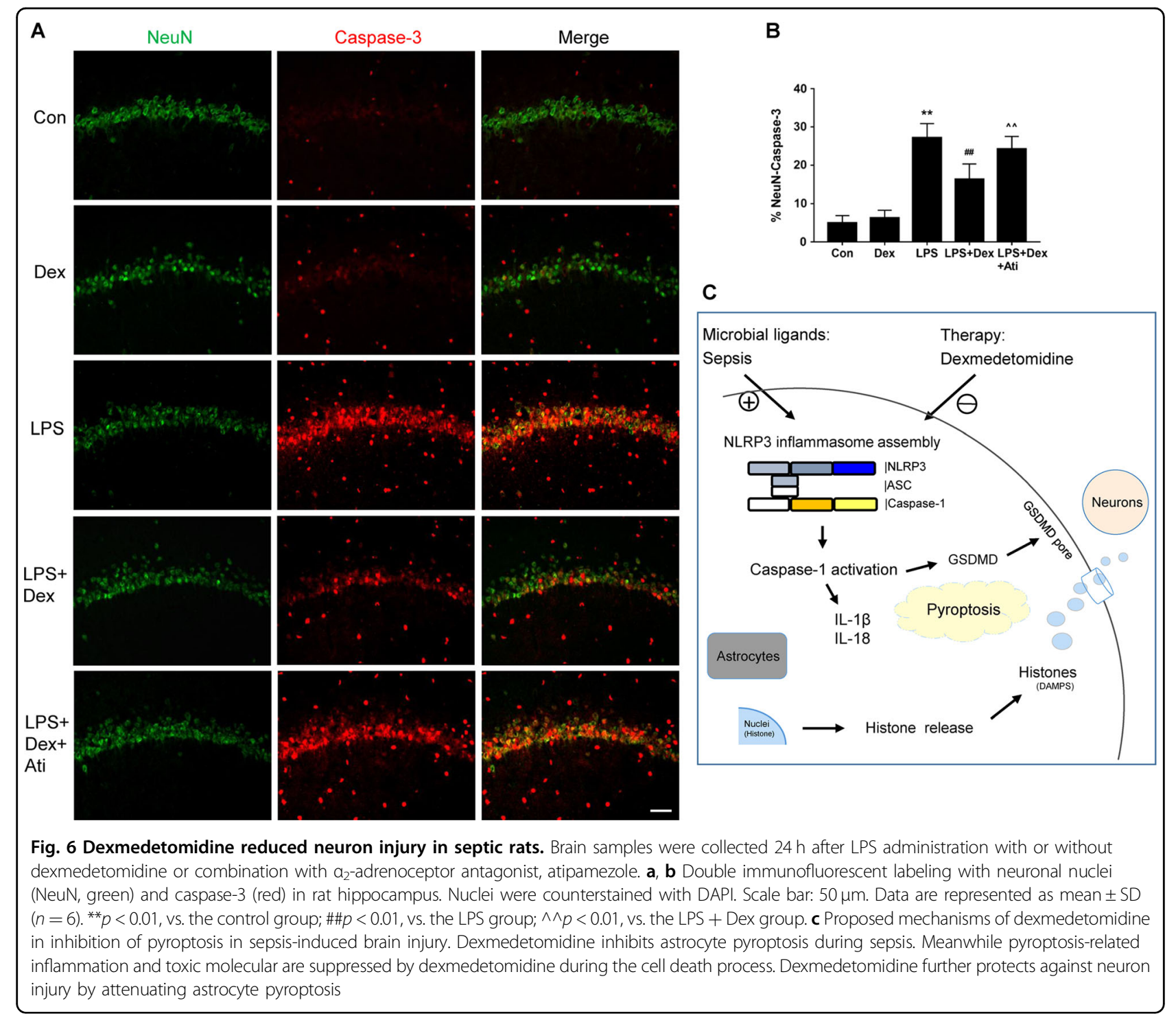

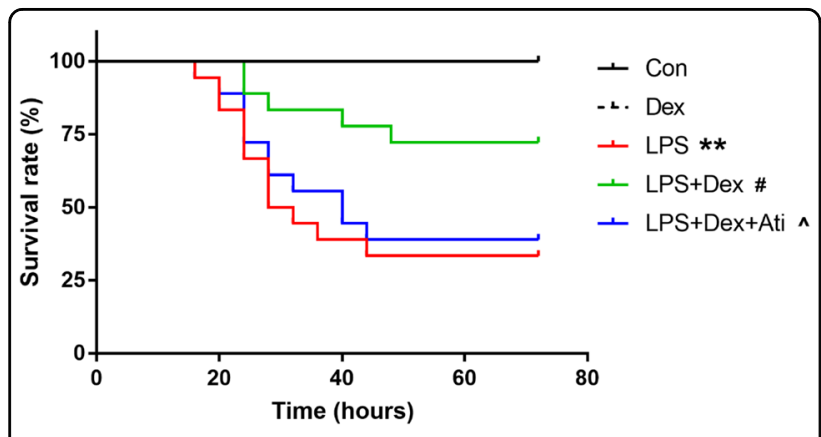

Fig. 7 Dexmedetomidine improved survival rate in septic rats. Survival rate within $72 \mathrm{~h}$ was analyzed after LPS administration by Kaplan-Meier log-rank test $(n=12-18) .{ }^{* *} p<0.01$, vs. the control group; $\# p<0.05$, vs. the LPS group; $\wedge p<0.05$, vs. the LPS + Dex group pyroptosis, and ferroptosis ${ }^{35}$, and act as damageassociated molecular patterns (DAMPs) molecules resulting in further cytotoxicity to surrounding cells. This process has been implicated in sepsis, organ injury and neurodegenerative disease ${ }^{36,37}$. It has been demonstrated the toxic effects of extracellular histone release, which result in endothelial dysfunction, organ failure, and death during sepsis ${ }^{37}$. LPS-induced acute kidney injury in mice partially involves extracellular histones, as indicated by the observation that administration of histoneneutralizing antibodies prevents kidney dysfunction ${ }^{38}$. Exogenous histone administration increases brain infarct volume, whilst anti-H2A/H4 antibody decreases the injury, indicating the possible detrimental effects of extracellular histone in ischemic stroke $\mathrm{e}^{39}$. Our data demonstrated that histone relocation and release were 
detectable after LPS treatment, whilst dexmedetomidine restricted histone within the nuclei and decreased histone in the extracellular space. Furthermore, exogenic histones caused neuronal loss, thus indicating that dexmedetomidine further indirectly protected neurons from the cytotoxicity associated with extracellular histones. These data suggest that the release of histones may be exacerbated in LPS-induced pyroptosis, consistent with previous findings which indicate that histones are present in the surrounding extracellular milieu after some forms of cell death, including pyroptosis ${ }^{35}$. Dexmedetomidine may exert its neuroprotective effects by decreasing cytotoxic extracellular histone release during this process.

In this study, we found that dexmedetomidine increased cell survival, and suppressed inflammasome activation and GSDMD augmentation in LPS-induced pyroptotic cell death in astrocytes, indicating that dexmedetomidine was involved in ameliorating astrocyte pyroptosis. This is in line with a recent finding that dexmedetomidine attenuates subarachnoid hemorrhage-associated brain injury by inhibiting the NLRP3 inflammasome pathway ${ }^{40}$.

Although dexmedetomidine protected neurons has been well demonstrated in various models of brain inju$\operatorname{ries}^{22,24,41,42}$, its protective effect on astrocytes has not been reported yet. Astrocytes are an important immunological component of the central nervous system (CNS), participating in synaptic plasticity and information processing in the neuronal circuit. They exert metabolic effects and provide structural support for neurons, and also play a role in modulating neuron homeostasis and higher neuronal functions. The interaction between astrocytes and neurons plays a crucial role in the development and progression of diverse neurological disorders. Alterations in the physiological function of astrocyte induce the loss of essential neurosupportive and neuroprotective functions, resulting in several cerebral disorders $^{43}$. Microglia, as the second most abundant glia cell types in the $\mathrm{CNS}^{44}$, are activated quickly under pathologic stimuli, e.g. LPS, which, in turn, release various proinflammatory mediators within the CNS, leading to highly "inflammable" inflammatory state. In addition, microglia are activated earlier than astrocytes and facilitate astrocyte activation. Subsequently, activated astrocytes in turn modulate activities of microglia and also promote activation of distant microglia ${ }^{45}$. They eventually "collaborate" with each other and enhance inflammatory responses in the pathological conditions such as sepsiscaused neuroinflammation and finally result in neuronal injury and brain functional impairment. Moreover, previous studies demonstrated that over-activated astrocytes themselves could eventually undergo cell death in presence of different insults ${ }^{6,46}$, and those astrocytes were unable to provide their homeostatic function and protect neurons; all of which contributed to the development of the deleterious neurological sequelae $e^{6,47}$. Therefore, as the first line of CNS defense, it is important to protect astrocytes from pathological insults in order to further protect neurocentric brain function. Furthermore, peripheral LPS administration may trigger inflammasome activation and the production of inflammatory mediators within the brain ${ }^{48}$. Studies have demonstrated that the development of neuroinflammation-related brain disorders is associated with a significant increase in caspase$1^{17}$. Caspase- 1 , a core feature of pyroptosis, is activated during pyroptosis, initiating the release of mature IL-1 $\beta$ and IL-18. Prolonged elevation of IL-1 $\beta$ and IL-18 causes neurotoxicity ${ }^{49,50}$; and high concentrations of IL- $1 \beta$ and IL-18 have been demonstrated in the cerebrospinal fluid (CSF) and brain tissue of patients with CNS infection, brain injury, and neurodegenerative disorders ${ }^{51-53}$. In this study, we observed an elevated level of caspase- 1 immunoreactivity in astrocyte cells, as well as an increase in IL$1 \beta$ and IL-18 release and histone production in the brain of septic rats. Furthermore, neuronal injury was observed following LPS administration, indicating that pyroptosis may play a vital role in the development of neuroinflammation and sepsis-induced brain injury, whilst impaired astrocytes failed to effectively provide neuronal support. Dexmedetomidine abolished the above detrimental changes and protected both glia and neurons and ultimately preserved brain functions per se. Indeed, it reduced coma free time when used in ICU for septic patients ${ }^{54}$.

It is worth mentioning that except attenuating inflammation and protecting glia cells and neurons afforded by dexmedetomidine as discussed above, its organ protections in particular on brain, kidney, and lung were well documented previously ${ }^{12,22,42,55-60}$. In addition, dexmedetomidine can promote natural sleep whilst natural sleep is important in maintaining physiological homeostasis including preserving immunofunction and restoring body energy and repairing potential inherent injury ${ }^{61-64}$. Owning to all above but not limited favorable effects of dexmedetomidine, it effectively decreased the mortality induced by LPS found in the current study which was in line with our previous report ${ }^{65}$.

There are indeed some limitations. Although the dexmedetomidine concentrations used in our experiment have been repeatedly reported previously ${ }^{22,57,60,66}$, it cannot be denied that there is still a gap between laboratory study and clinical practice. It has been shown that the therapeutic concentration of dexmedetomidine in the human plasma can be reached up to $1 \mu \mathrm{M}$ after intravenous infusion ${ }^{67}$. It was also reported that $25 \mu \mathrm{g} / \mathrm{kg}$ dexmedetomidine provided significant neuroprotective effect against isoflurane-induced injury in the young ${ }^{22}$. Those work may arguably provide a basis of the doses of dexmedetomidine that were chosen for our in vitro and 
in vivo study, respectively. However, it must be admitted that the dose of dexmedetomidine is considerably higher than the dose used clinically. Once can appreciate is that drug used in animals in general is normally 7-10 times higher than used in humans.

Collectively, to our acknowledge, this study demonstrated, for the first time, that dexmedetomidine protected against pyroptosis in sepsis-induced brain injury. These data suggest that dexmedetomidine may enhance neuronal survival by inhibiting astrocyte pyroptosis and, therefore, decrease potentially harmful pyroptosis-related inflammatory responses within brain, indicating a novel neuroprotective mechanism of dexmedetomidine in sepsis-induced brain injury.

\section{Materials and methods}

\section{Cell lines and cell treatments}

Human astrocyte $1321 \mathrm{~N} 1$ cells and rat neuron PC12 cells were purchased from the European Collection of Authenticated Cell Cultures (ECACC, Porton Down, UK). 1321N1 cells were cultured in Dulbecco's modified Eagle's Medium (DMEM) (Gibco; Invitrogen, USA) supplemented with $10 \%$ fetal bovine serum and $100 \mathrm{U} / \mathrm{ml}$ penicillin-streptomycin. Some cohort 1321N1 cultures were treated with lipopolysaccharide (LPS) E. coli O111: B4 $(100 \mathrm{ng} / \mathrm{ml}$, Sigma-Aldrich, Poole, UK) for $24 \mathrm{~h}$, some cohort cultures were treated with $1 \mu \mathrm{M}$ dexmedetomidine (Sigma-Aldrich) 30 min before LPS treatment ${ }^{22}$, and some cohort cultures were treated with TNF- $\alpha(100 \mathrm{ng} / \mathrm{ml}$, Sigma-Aldrich) for $24 \mathrm{~h}$. PC12 cells were maintained in RPMI-1640 medium (Gibco; Invitrogen, USA) containing $10 \%$ heat-inactivated horse serum, $5 \%$ fetal bovine serum and $100 \mathrm{U} / \mathrm{ml}$ penicillin-streptomycin. To induce the differentiation of PC12 cells into neuronal like cells, 50 $\mathrm{ng} / \mathrm{ml}$ nerve growth factor (NGF) was added into the medium with $1 \%$ horse serum and cells were seeded on a Collagen IV coated dish for $24 \mathrm{~h}$. The medium was routinely replaced with fresh medium every 2 days for 5 days until neuronal differentiation had taken place. PC12 cells were treated with calf thymus histones $(1000 \mathrm{ng} / \mathrm{ml}$, Sigma-Aldrich) for $24 \mathrm{~h}$. The cells were incubated at $37^{\circ} \mathrm{C}$ in a humidified atmosphere with $5 \% \mathrm{CO}_{2}$.

\section{Animals and drug administration}

The experimental procedures were approved by the Peking University Biomedical Ethics Committee experimental animal ethics branch (Approval No. J201807) and performed in accordance with the institutional guidelines. Adult male Sprague-Dawley rats $(250-300 \mathrm{~g})$ were purchased from the Experimental Animal Center of Peking University Health Center (Beijing, China). Rats were housed in standardized conditions with room temperature $24 \pm 2{ }^{\circ} \mathrm{C}$, controlled humidity $55 \pm 5 \%, 12 \mathrm{~h}$ light $/ 12$ $\mathrm{h}$ dark cycle and access to water ad libitum. Rats were injected intraperitoneally with $10 \mathrm{mg} / \mathrm{kg}$ LPS (SigmaAldrich), dissolved and diluted in sterile normal saline. Dexmedetomidine (Sigma-Aldrich) was then intraperitoneally injected at a dose of $25 \mu \mathrm{g} / \mathrm{kg}$ every $2 \mathrm{~h}$ for three times immediately after LPS administration ${ }^{22}$. One cohort was injected intraperitoneally with $500 \mu \mathrm{g} / \mathrm{kg} \alpha_{2}$-adrenoceptor antagonist atipamezole (Sigma-Aldrich) every $2 \mathrm{~h}$ in three doses prior to the administration of dexmedetomidine ${ }^{22}$. Controls received comparable volume injections of saline. At $24 \mathrm{~h}$ after the first treatment, animals were sacrificed with pentobarbitone $(100 \mathrm{mg} / \mathrm{kg}$, i.p.). Their hippocampus was quickly dissected and snap frozen in liquid nitrogen from the half of the animals from each group. The remaining animals were perfused with $4 \%$ paraformaldehyde and their brains were collected.

\section{Flow cytometry}

Propidium iodide (PI; Sigma-Aldrich) staining was used to measure cellular death. Cells were collected in a fluorescence-activated cell sorting (FACS) tube and washed twice before resuspension in FACS buffer. PI was added to make the final concentration to $1 \mu \mathrm{g} / \mathrm{ml}$ and incubated in the dark for $5 \mathrm{~min}$ at room temperature. PI fluorescence was detected with flow cytometry. Each assay included at least 10,000 gated events.

\section{Western blot analysis}

The lysates from cultured cells were centrifuged, the supernatant was collected, and total protein concentration was quantified by the Bradford protein assay (Bio-Rad, Hemel Hempstead, UK). The protein extracts $(80 \mu \mathrm{g}$ per sample) underwent SDS-polyacrylamide gel electrophoresis and were then transferred to a polyvinylidene difluoride membrane. The membranes were blocked with $5 \%$ non-fat dried milk and probed with primary antibodies: anti-NLRP3 (1:1000, Cell Signaling Technology), anti-ASC (1:500, Santa Cruz), anti-caspase-1 (1:1000, Abcam) and anti-GSDMD (1:1000, Novus Biologicals) in TBS-T overnight at $4{ }^{\circ} \mathrm{C}$, followed by HRP-conjugated secondary antibody for $1 \mathrm{~h}$. The loading control was GAPDH (1:10,000, Millipore). The blots were detected with enhanced chemiluminescence (ECL) system (Santa Cruz Biotechnology) and analyzed with GeneSnap (Syngene, Cambridge, UK). The protein band intensity was normalized with GAPDH and expressed as ratio of the control.

\section{Immunofluorescent staining}

For in vitro fluorescent staining, cells were fixed in $4 \%$ paraformaldehyde in $0.1 \mathrm{~mol} / \mathrm{L}$ PBS solution, then blocked with $10 \%$ normal goat serum for $1 \mathrm{~h}$ and incubated with the primary antibodies: rabbit anti-caspase-1 (1:200, Abcam), mouse anti-ASC (1:200, Santa Cruz), rabbit anti-histone $\mathrm{H} 4$ (1:200, Abcam), mouse anti-F-actin 
(1:200, Abcam) overnight followed by fluorescently conjugated secondary antibodies for $1 \mathrm{~h}$. For in vivo fluorescent staining, brains were collected and fixed in $4 \%$ paraformaldehyde for $16 \mathrm{~h}$ at $4{ }^{\circ} \mathrm{C}$, followed by dehydration in $30 \%$ sucrose solution for $24 \mathrm{~h}$ at $4{ }^{\circ} \mathrm{C}$. Brain samples were then cryosectioned at $-20^{\circ} \mathrm{C}$ into $14 \mu \mathrm{M}$ slices and mounted onto slides. Sections were rinsed in $0.1 \%$ Triton in PBS and incubated with $10 \%$ normal goat serum. Sections were then incubated overnight with rabbit anti-caspase-1 (1:200, Abcam), mouse anti-glial fibrillary acidic protein (GFAP) (1:200, Sigma-Aldrich), rabbit anti-caspase-3 (1:200, Cell Signaling Technology), mouse anti-neuronal nuclei (NeuN) (1:200, Millipore), followed by secondary antibodies. For double-labeled immunofluorescence, cells and tissue samples were incubated with the first primary antibody overnight, followed by the first secondary antibody, and then the second primary antibody and the second secondary antibody. The slides were counterstained with nuclear dye 4',6diamidino-2-phenylindole (DAPI) and mounted with Vectashield mounting medium (Vector Laboratories, USA). Ten high-power fields at $\times 20$ magnification were photographed using an AxioCam digital camera (Zeiss, Welwyn Garden City, UK) mounted on an Olympus BX60 microscope (Olympus, Middlesex, UK) with Zeiss KS-300 software (Zeiss, Welwyn Garden City, UK).

\section{Hoechst and PI staining}

PC12 cells were co-stained with Hoechst $33342(1 \mu \mathrm{g} /$ $\mathrm{ml}$, Sigma-Aldrich) and PI $(5 \mu \mathrm{g} / \mathrm{ml}$, Sigma-Aldrich) for $20 \mathrm{~min}$ at room temperature, then stained nuclei were observed under a fluorescent microscope ${ }^{68}$. Cell death was identified on the basis of positive staining with PI and apoptotic nuclear morphology changes with Hoechst dye.

\section{Enzyme-linked immunosorbent assay}

The cell medium histone H4, brain tissue IL-1 $\beta$, IL-18 (Neobioscience Technology, Shenzhen, China) and histone H4 (USCN Life Science, Wuhan, China) concentrations were measured by using enzyme-linked immunosorbent assay (ELISA) kits following the manufacturer's instructions.

\section{Survival analysis}

Rats receiving the same interventions as indicated were maintained under normal housing conditions. They were monitored every $4 \mathrm{~h}$ and killed once they reached humane endpoints (significant body weight loss, immobilized, very poor grooming, and back arching). The survival curve was constructed.

\section{Statistical analysis}

The statistical analyses were performed with SPSS version 20.0. Data were expressed as mean \pm SD and analyzed by one-way analysis of variance (ANOVA) with Bonferroni's post hoc test or Student's $t$-test. A $p$-value $<0.05$ was considered to be statistically significant. Animal survival analysis was performed using Kaplan-Meier survival estimates, and statistical significance was analyzed by the log-rank test (GraphPad Prism 5.0; GraphPad Software).

\section{Acknowledgements}

This study was supported by grants from the Wu Jieping Medical Foundation, Beijing, China (320.6750.15175), the Chinese Society of Cardiothoracic and Vascular Anesthesiology, Beijing, China and Beijing Municipal Natural Science Foundation, Beijing, China (7154245), the BOC chair grant, the Westminster Medical School Research Trust and BJA/RCoA project grant. The study sponsors had no role in study design, in the collection, analysis, and interpretation of data, or in the writing of the report. The contents of this article are solely the responsibility of the authors and do not necessarily represent the official views of all the funding bodies. Y.B.S. received a scholarship (201503780064) from China Scholarship Council, Beijing, China.

\section{Author details}

${ }^{1}$ Department of Anesthesiology and Critical Care Medicine, Peking University First Hospital, Beijing, China. ${ }^{2}$ Anaesthetics, Pain Medicine and Intensive Care, Department of Surgery and Cancer, Faculty of Medicine, Imperial College London, Chelsea and Westminster Hospital, London, UK. ${ }^{3}$ Department of Obstetrics and Gynecology, the Second Affiliated Hospital of Wenzhou Medical University, Wenzhou, Zhejiang Province, China

\section{Conflict of interest}

The authors declare that they have no conflict of interest.

\section{Publisher's note}

Springer Nature remains neutral with regard to jurisdictional claims in published maps and institutional affiliations.

Received: 15 November 2018 Revised: 30 January 2019 Accepted: 1 February 2019

Published online: 18 February 2019

\section{References}

1. Schieveld, J. N. M., van Tuijl, S. \& Pikhard, T. On nontraumatic brain injury in pediatric critical illness, neuropsychologic short-term outcome, delirium, and resilience. Crit. Care Med. 41, 1160-1161 (2013).

2. Jackson, A., Gilbert, J., Young, G. \& Bolton, C. The encephalopathy of sepsis. Can. J. Neurol. Sci. 12, 303-307 (1985)

3. Lee, J. W. et al. Neuro-inflammation induced by lipopolysaccharide causes cognitive impairment through enhancement of beta-amyloid generation. J. Neuroinflamm. 5, 37 (2008).

4. Weberpals, M. et al. NOS2 gene deficiency protects from sepsis-induced longterm cognitive deficits. J. Neurosci. 29, 14177-14184 (2009).

5. Rama Rao, K. V. \& Tammy, K. Neuron-astrocyte interactions in neurodegenerative diseases: role of neuroinflammation. Clin. Exp. Neuroimmunol. 6, 245-263 (2015).

6. Sharma, A., Patro, N. \& Patro, I. K. Lipopolysaccharide-induced apoptosis of astrocytes: therapeutic intervention by minocycline. Cell Mol. Neurobiol. 36, 577-592 (2016).

7. Fan, L. et al. Systemic inflammation induces a profound long term brain cell injury in rats. Acta Neurobiol. Exp. 74, 298-306 (2014).

8. Reade, M. C., Eastwood, G. M. \& Bellomo, R. Effect of dexmedetomidine added to standard care on ventilator-free time in patients with agitated delirium: a randomized clinical trial. JAMA 315, 1460-1468 (2016).

9. Barr, J. et al. Clinical practice guidelines for the management of pain, agitation, and delirium in adult patients in the intensive care unit. Crit. Care Med. 41, 263-306 (2013).

10. Zhang, D.-F. et al. Impact of dexmedetomidine on long-term outcomes after noncardiac surgery in elderly: 3-year follow-up of a randomized controlled trial. Ann. Surg. https://doi.org/10.1097/SLA.0000000000002801 (2018). 
11. Kong, W. et al. Dexmedetomidine alleviates LPS-induced septic cardiomyopathy via the cholinergic anti-inflammatory pathway in mice. Am. J. Transl. Res. 9, 5040-5047 (2017).

12. Cui, J., Zhao, H., Lu, K., Yi, B. \& Ma, D. Dexmedetomidine attenuates bilirubininduced lung alveolar epithelial cell death in vitro and in vivo. Crit. Care Med. 43, e356-e368 (2015)

13. Luo, C. et al. Dexmedetomidine protects mouse brain from ischemiareperfusion injury via inhibiting neuronal autophagy through up-regulating HIF-1alpha. Front. Cell Neurosci. 11, 1-13 (2017).

14. Guo, H., Callaway, J. B. \& Ting, J. P.-Y. Inflammasomes: mechanism of action, role in disease, and therapeutics. Nat. Med. 21, 677-687 (2015),

15. Cervantes, J., Nagata, T., Uchijima, M., Shibata, K. \& Koide, Y. Intracytosolic Listeria monocytogenes induces cell death through caspase-1 activation in murine macrophages. Cell Microbiol. 10, 41-52 (2008).

16. Bergsbaken, T., Fink, S. L. \& Cookson, B. T. Pyroptosis: host cell death and inflammation. Nat. Rev. Microbiol. 7, 99-109 (2009).

17. Mamik, M. K. \& Power, C. Inflammasomes in neurological diseases: emerging pathogenic and therapeutic concepts. Brain 140, 2273-2285 (2017).

18. G. Walsh, J., A. Muruve, D. \& Power, C. Inflammasomes in the CNS. Nat. Rev. Neurosci. 15, 84-97 (2014).

19. Song, L., Pei, L., Yao, S., Wu, Y. \& Shang, Y. NLRP3 inflammasome in neurological diseases, from functions to therapies. Front. Cell Neurosci. 11, 1-17 (2017).

20. Lv, M., Zeng, H., He, Y., Zhang, J. \& Tan, G. Dexmedetomidine promotes liver regeneration in mice after $70 \%$ partial hepatectomy by suppressing NLRP3 inflammasome not TLR4/NFkappaB. Int. Immunopharmacol. 54, 46-51 (2018).

21. Li, Y. et al. Dexmedetomidine attenuates pancreatic injury and inflammatory response in mice with pancreatitis by possible reduction of NLRP3 activation and up-regulation of NET expression. Biochem. Biophys. Res. Commun. 495 2439-2447 (2018).

22. Sanders, R. D. et al. Dexmedetomidine attenuates isoflurane-induced neurocognitive impairment in neonatal rats. Anesthesiology 110, 1077-1085 (2009).

23. Wang, Z. et al. Effects of propofol-dexmedetomidine combination on ischemia reperfusion-induced cerebral injury. NeuroRehabilitation 35, 825-834 (2014).

24. Degos, V. et al. Neuroprotective effects of dexmedetomidine against glutamate agonist-induced neuronal cell death are related to increased astrocyte brain-derived neurotrophic factor expression. Anesthesiology 118, 1123-1132 (2013).

25. Matsuda, A. et al. Novel therapeutic targets for sepsis: regulation of exaggerated inflammatory responses. J. Nippon Med. Sch. 79, 4-18 (2012).

26. Aziz, M., Jacob, A., Yang, W.-L., Matsuda, A. \& Wang, P. Current trends in inflammatory and immunomodulatory mediators in sepsis. J. Leukoc. Biol. 93, 329-342 (2013)

27. Zou, L. et al. Imaging lymphoid cell death in vivo during polymicrobial sepsis. Crit. Care Med. 43, 2303-2312 (2015).

28. Jorgensen, I. \& Miao, E. A. Pyroptotic cell death defends against intracellular pathogens. Immunol. Rev. 265, 130-142 (2015).

29. Aziz, M., Jacob, A. \& Wang, P. Revisiting caspases in sepsis. Cell Death Dis. 5, e1526 (2014).

30. Garib, F. Y., Rizopulu, A. P., Kuchmiy, A. A. \& Garib, V. F. Inactivation of inflammasomes by pathogens regulates inflammation. Biochemistry $\mathbf{8 1}$, 1326-1339 (2016).

31. Croker, B. A., O'Donnell, J. A. \& Gerlic, M. Pyroptotic death storms and cytopenia. Curr. Opin. Immunol. 26, 128-137 (2014).

32. Shi, J. et al. Cleavage of GSDMD by inflammatory caspases determines pyroptotic cell death. Nature 526, 660-665 (2015).

33. Sborgi, L. et al. GSDMD membrane pore formation constitutes the mechanism of pyroptotic cell death. EMBO J. 35, 1766-1778 (2016).

34. Hotchkiss, R. S., Strasser, A., McDunn, J. E. \& Swanson, P. E. Cell death. N. Engl. J. Med. 361, 1570-1583 (2009).

35. Bird, A. Perceptions of epigenetics. Nature 447, 396-398 (2007).

36. Chaput, C. \& Zychlinsky, A. Sepsis: the dark side of histones. Nat. Med. 15, 1245-1246 (2009)

37. $\mathrm{Xu}, \mathrm{J}$. et al. Extracellular histones are major mediators of death in sepsis. Nat. Med. 15, 1318-1321 (2009).

38. Allam, R. et al. Histones from dying renal cells aggravate kidney injury via TLR2 and TLR4. J. Am. Soc. Nephrol. 23, 1375-1388 (2012).
39. De Meyer, S. F., Suidan, G. L., Fuchs, T. A., Monestier, M. \& Wagner, D. D Extracellular chromatin is an important mediator of ischemic stroke in mice. Arterioscler. Thromb. Vasc. Biol. 32, 1884-1891 (2012).

40. Yin, D. et al. Dexmedetomidine attenuated early brain injury in rats with subarachnoid haemorrhage by suppressing the inflammatory response: the tlr4/nf-jb pathway and the nlrp3 inflammasome may be involved in the mechanism. Brain Res. 1698, 1-10 (2018).

41. Hu, J. et al. Dexmedetomidine prevents cognitive decline by enhancing resolution of high mobility group box 1 protein-induced inflammation through a vagomimetic action in mice. Anesthesiology 128, $921-931$ (2018).

42. Alam, A. et al. Neuroprotection and neurotoxicity in the developing brain: an update on the effects of dexmedetomidine and xenon. Neurotoxicol. Teratol. 60, 102-116 (2017)

43. Dossi, E., Vasile, F. \& Rouach, N. Human astrocytes in the diseased brain. Brain Res. Bull. 136, 139-156 (2018).

44. Ransohoff, R. M. \& Perry, V. H. Microglial physiology: unique stimuli, specialized responses. Annu. Rev. Immunol. 27, 119-145 (2009).

45. Liu, W., Tang, Y. \& Feng, J. Cross talk between activation of microglia and astrocytes in pathological conditions in the central nervous system. Life Sci. 89, 141-146 (2011).

46. Alfonso-Loeches, S., Ureña-Peralta, J. R., Morillo-Bargues, M. J., Oliver-De La Cruz, J. \& Guerri, C. Role of mitochondria ROS generation in ethanol-induced NLRP3 inflammasome activation and cell death in astroglial cells. Front. Cell Neurosci. 8, 216 (2014)

47. Allaman, I., Belanger, M. \& Magistretti, P. J. Astrocyte-neuron metabolic relationships: for better and for worse. Trends Neurosci. 34, 76-87 (2011).

48. Jiang, P. et al. Salvianolic acid B protects against lipopolysaccharide-induced behavioral deficits and neuroinflammatory response: involvement of autophagy and NLRP3 inflammasome. J. Neuroinflamm. 14, 239 (2017).

49. Lee, P. R. et al. Protease-activated receptor-1 activation by granzyme B causes neurotoxicity that is augmented by interleukin-1 beta. J. Neuroinflamm. 14, 131 (2017).

50. Hein, A. M. et al. Sustained hippocampal IL-1beta overexpression impairs contextual and spatial memory in transgenic mice. Brain Behav. Immun. 24, 243-253 (2010)

51. Huang, W.-X., Huang, P. \& Hillert, J. Increased expression of caspase-1 and interleukin-18 in peripheral blood mononuclear cells in patients with multiple sclerosis. Mult. Scler. 10, 482-487 (2004).

52. Licastro, F. et al. Increased plasma levels of interleukin-1, interleukin-6 and a-1antichymotrypsin in patients with Alzheimer's disease: peripheral inflammation or signals from the brain? J. Neuroimmunol. 103, 97-102 (2000).

53. de Jong, B. A. et al. Production of $\mathrm{IL}-1 \mathrm{~b}$ and IL-1Ra as risk factors for susceptibility and progression of relapse-onset multiple sclerosis. J. Neuroimmunol. 126, 172-179 (2002).

54. Riker, R. R. et al. Dexmedetomidine vs midazolam for sedation of critically ill patients. JAMA 301, 489-499 (2009).

55. Ma, J. et al. Dexmedetomidine-mediated prevention of renal ischemiareperfusion injury depends in part on cholinergic anti-inflammatory mechanisms. Anesth. Analg. https://doi.org/10.1213/ANE.0000000000003820 (2018).

56. Cui, J. et al. Dexmedetomidine attenuates oxidative stress induced lung alveolar epithelial cell apoptosis in vitro. Oxid. Med. Cell Longev. 2015, 358396 (2015).

57. Gu, J. et al. Dexmedetomidine provides renoprotection against ischemiareperfusion injury in mice. Crit. Care. 15, R153 (2011).

58. Sanders, R. D. et al. Dexmedetomidine provides cortical neuroprotection: impact on anaesthetic-induced neuroapoptosis in the rat developing brain. Acta Anaesthesiol. Scand. 54, 710-716 (2010).

59. Rajakumaraswamy, N. et al. Neuroprotective interaction produced by xenon and dexmedetomidine on in vitro and in vivo neuronal injury models. Neurosci. Lett. 409, 128-133 (2006)

60. Ma, D. et al. Dexmedetomidine produces its neuroprotective effect via the alpha 2A-adrenoceptor subtype. Eur. J. Pharmacol. 502, 87-97 (2004).

61. $\mathrm{Wu}, \mathrm{X}$.-H. et al. Low-dose dexmedetomidine improves sleep quality pattern in elderly patients after noncardiac surgery in the intensive care unit: a pilot randomized controlled trial. Anesthesiology 125, 979-991 (2016).

62. Xie, L. et al. Sleep drives metabolite clearance from the adult brain. Science 342, 373-377 (2013)

63. Besedovsky, L., Lange, T. \& Born, J. Sleep and immune function. Pflug. Arch 463, 121-137 (2012). 
64. Everson, C. A., Henchen, C. J., Szabo, A. \& Hogg, N. Cell injury and repair resulting from sleep loss and sleep recovery in laboratory rats. Sleep 37, 1929-1940 (2014).

65. Qiao, H., Sanders, R. D., Ma, D., Wu, X. \& Maze, M. Sedation improves early outcome in severely septic Sprague Dawley rats. Crit. Care. 13, R136 (2009).

66. Dahmani, S., Rouelle, D., Gressens, P. \& Mantz, J. Characterization of the postconditioning effect of dexmedetomidine in mouse organotypic hippocampal slice cultures exposed to oxygen and glucose deprivation Anesthesiology 112, 373-383 (2010).

67. Venn, R. M., Karol, M. D. \& Grounds, R. M. Pharmacokinetics of dexmedetomidine infusions for sedation of postoperative patients requiring intensive caret. Br. J. Anaesth. 88, 669-675 (2002).

68. Huang, C. et al. Shikonin kills glioma cells through necroptosis mediated by RIP-1. PLOS ONE 8, e66326 (2013). 